\title{
Malignant glaucoma
}

\author{
RICHARD J. SIMMONS \\ Boston, Mass., U.S.A.
}

My interest in malignant glaucoma stems from my practice in association with Dr. Paul A. Chandler and our work in cooperation with Dr. W. Morton Grant, and I am speaking not as an individual but as a member of a team of three.

A review of our files revealed 66 patients with some form of malignant glaucoma in one or both eyes. Of these 66 patients, $3^{2}$ had typical full-blown phakic malignant glaucoma, and the remaining 34 included aphakic cases, some with a milder and more transient form of the disease, as well as some with complex ocular pathology in which malignant glaucoma was included as one of several diagnoses.

\section{CLASSICAL MALIGNANT GLAUGOMA}

Since it was first described by von Graefe (1 869), malignant glaucoma has been recognized as one of the most serious complications of operation for angle-closure glaucoma. It has occurred in cases of acute, sub-acute, and chronic angle-closure glaucoma, regardless of the type of operation used, but it is fortunately rare and has been reported in the literature as occurring in only 2 to 4 per cent. of patients operated on for angle-closure glaucoma.

Classical malignant glaucoma* is characterized by shallowing or flattening of the anterior chamber and elevation of tension after the operation for angle-closure glaucoma despite the presence of an open coloboma of the iris. The tension may be normal at first but eventually becomes elevated. The time of onset varies; it may occur immediately after surgery or a year or more later. Often the onset coincides with the cessation of cycloplegic drops after surgery or with the institution of miotic drops (Chandler and Grant, 1962). Characteristically malignant glaucoma shows a worsening course when treated by conventional glaucoma therapy (Chandler, Simmons, and Grant, r968), and the fellow eye shows a marked disposition to develop the same malignant course. If the same conditions, such as closure of the angle after an attack of angle-closure glaucoma, exist at the time of surgery in the fellow eye, a malignant course can be expected. It may persist in eyes in which the lens has been extracted with the intention of counteracting the malignant course. In such aphakic eyes, the features and behaviour of the malignant glaucoma is similar to that in phakic eyes.

Besides these classical features of malignant glaucoma, we have observed certain other characteristics.

In our cases of primary angle-closure glaucoma followed by malignant glaucoma, partial or total closure of the angle at the time of operation has been a constant feature. When the angle has been totally open at the time of surgery, malignant glaucoma has not followed. The significance of this observation is as yet unknown. The level of tension at the time of surgery has not been a good guide in our experience to the likelihood of a malignant course. The tension may be normal or sub-normal from the effects of acetazola- 
mide and glycerol, or through the suppression of aqueous formation in an attack of angleclosure glaucoma, and yet a malignant course may follow.

We have also observed interesting features regarding the relationship between the $\vec{F}$ anterior vitreous, the ciliary processes, and the peripheral lens. The tips of the ciliaryo processes may touch the lens as viewed through iris colobomata. The ciliary processes are $\frac{\bar{O}}{\overline{\underline{T}}}$ frequently rotated anteriorly, their tips are sometimes flattened against the lens, and in one $\frac{\omega^{n}}{\vec{D}}$ case one of us (W.M.G.) observed some of the tips of the ciliary processes firmly adherent $\stackrel{\mathbb{D}}{\circ}$ to the lens by synechiae after an attack of malignant glaucoma had subsided. The space between the ciliary processes, however, can be seen to be open, and through the spaces one $\vec{\circ}$ can see the vitreous behind. In phakic eyes with malignant glaucoma we have seen the anterior vitreous face to be abnormally forward behind the ciliary processes, and in aphakic eyes we have observed it touching and/or adherent to the ciliary processes. We have observed these features regularly in cases of malignant glaucoma and we have taught $\overparen{G}$ that they are characteristic of the condition.

Slit-lamp examination of the vitreous of certain eyes, both phakic and aphakic, with $\stackrel{N}{\mathscr{G}}$ malignant glaucoma has revealed optically clear areas within the vitreous cavity (which $\frac{9}{2}$ we have interpreted as pockets of fluid). In a few cases in which the eye was unusually clear, a smooth vitreous face has been seen in the eye anterior to the middle of the vitreous cavity. (We have interpreted this as the posterior hyaloid.) The vitreous face has been $\stackrel{\bigcirc}{\mathcal{S}}$ smooth and seemingly bowed forward and an optically clear space has been present $\vec{c}$ behind it.

MEDIGAL TREATMENT OF MALIGNANT GLAUCOMA

Before the report by Chandler and Grant (1962) of the successful use of mydriatic-cycloplegic therapy, the medical treatment of malignant glaucoma was uniformly ineffective. ڤ̊ Miotics were frequently used with no response or with an actual worsening of the malignant process. Usually in frustration the physician gave more and more miotics and the malignant course continued relentlessly.

Chandler and Grant (1962) developed the idea of mydriatic-cycloplegic therapy from observations that malignant glaucoma frequently appeared after operations for angle- 0 closure glaucoma when mydriatic-cycloplegic therapy had been stopped postoperatively, or that it coincided with the institution of miotics for some residual glaucoma after the surgical procedure. In addition, they reasoned that the action of pilocarpine in tightening $ᄋ$ the ciliary muscle and loosening the zonule was undesirable in malignant glaucoma and that the opposite effect of cycloplegics in tightening the zonule might have a favourable action by pulling the lens backwards. Ghandler and Grant first employed this type of therapy in a series of eight cases, and it was successful in relieving the malignant glaucoma without surgery in all of them. However, with experience with further cases, it became of apparent that mydriatic-cycloplegic therapy would not relieve the malignant course in all cases.

By adding acetazolamide and using it concurrently with mydriatic-cycloplegic drops, medical treatment became effective in some of our patients in whom mydriatic-cycloplegic drops alone were ineffective (Chandler and others, 1968). We therefore use these $\stackrel{\mathscr{Q}}{+}$ agents in combination for the medical therapy of malignant glaucoma.

Tiberi and Diotallevi (1962) reported the successful use of hyperosmotic treatment $\frac{0}{\Phi}$ employing intravenous urea. Weiss, Shaffer, and Harrington (1963) reported the successful use of intravenous mannitol, and in the same year Offret, Valletta, and Coscas ( I963) reported the successful use of hexamethonium. Hyperosmotic agents and hexamethonium 
alone were primarily used to lower the pressure before removal of the lens. Frezzotti and Gentili (1964) demonstrated that hyperosmotic agents combined with mydriatic-cycloplegic therapy can produce beneficial effects not produced by either agent alone. They described two situations in which mydriatic-cycloplegic therapy and intravenous urea were each ineffective alone, but when they were used in combination the anterior chamber re-formed and thereafter remained under control with atropine therapy without surgery.

In view of this, we began the routine use of mydriatic-cycloplegic therapy plus a carbonic anhydrase inhibitor (acetazolamide) and hyperosmotic agents begun simultaneously and continued concurrently (Ghandler and others, I968). This combined therapy provides, we believe, the best medical regimen for malignant glaucoma at the present time. Specifically, it consists of the concurrent use of the following programme:

(r) Mydriatic-cycloplegic drops (Phenylephrine ro per cent., one drop four times a day, plus atropine I to 4 per cent., one drop four times a day).

(2) Acetazolamide $250 \mathrm{mg}$. by mouth, four times a day.

(3) Hyperosmotic agents by mouth or intravenously. For convenience we customarily use a solution of 50 per cent. glycerol by mouth, I ml./lb. body weight, once or twice a day as tolerated. The dose is taken within 5 minutes or less. As an alternative in the individual case for reasons of comfort and tolerance, mannitol $2 \mathrm{~g}$. $/ \mathrm{kg}$. body weight is administered intravenously once or twice a day.

We use this combined therapy for approximately 5 days, starting as soon as the diagnosis is made. If the anterior chamber has not re-formed on the fifth day, we then resort to surgery. In many patients the anterior chamber has begun to re-form in response to this medical therapy after only 2 or 3 days. In one case the anterior chamber re-formed after 5 days of combined therapy, and we have therefore chosen the fifth day as the time to abandon unsuccessful trials of medical therapy.

When the anterior chamber has been formed and the tension has been improved by this medical regimen, we gradually reduce the dosage. We first omit the hyperosmotic agents and then gradually reduce or omit the carbonic anhydrase inhibitors depending upon the level of tension. If there is significant closure of the anterior chamber angle because of synechiae and the control of tension is inadequate, the oral carbonic anhydrase inhibitors are continued on a long-term basis and topical epinephrine may be added. If the tension remains stable for some weeks we omit the phenylephrine drops. In some of our cases the malignant glaucoma returned when the cycloplegic (atropine) drops were stopped. In some cases it was possible to relieve it again by full combined medical regimen, but other cases did not respond to medical therapy a second time, and surgery was necessary. Topical atropine is therefore continued once daily indefinitely after the successful medical relief of malignant glaucoma. Long-term cycloplegic drops continued indefinitely once daily seem to be a small inconvenience compared to the greater risk of surgery.

After re-formation of the anterior chamber by medical therapy, we have avoided the future use of pilocarpine. The role of miotic therapy in aggravating and even precipitating malignant glaucoma has been repeatediy observed since the report of Chandler and Grant (1962). We do not know at present whether the use of miotics is permanently contraindicated after the medical relief of malignant glaucoma.

This medical regimen has as its goal not the temporary relief of the malignant glaucoma in readiness for lens extraction, but its permanent relief without surgery. We have found it to be successful in approximately 50 per cent. of our patients. In aphakic cases, the 
action of mydriatic-cycloplegic therapy in tightening the zonules is obviously of no value, but we have found medical therapy with carbonic anhydrase inhibitors and hyperosmotic $\stackrel{\text { o }}{.}$ agents similarly beneficial.

\section{SURGICAL TREATMENT OF MALIGNANT GLAUCOMA}

Since malignant glaucoma was first recognized, conventional glaucoma operations have been repeatedly employed, but like conventional medical therapy, they have uniformly failed.

Simple posterior sclerotomy was recommended by Weber (1877), who reported a case in $\overrightarrow{0}$ which he performed a posterior sclerotomy 8-10 mm. from the limbus and applied pressure $\vec{\longrightarrow}$ to the cornea for 2 minutes, and the anterior chamber re-formed. Other authors have $\omega_{\sigma}$ used this technique and some successes and a considerable number of failures have been $\frac{0}{0}$. reported. Others have used simple posterior sclerotomy with air injection with some in success $\dagger$. The use of simple posterior sclerotomy even immediately before lens extraction $\omega$ for malignant glaucoma fails to prevent a continuing malignant course. Therefore this $\hat{N}^{\circ}$ operation is inadequate.

Lens extraction has been widely used for malignant glaucoma since it was first suggested by Pagenstecher (1877) and used by Rheindorf (1887). Chandler (1950) reported the use of lens extraction in six cases and urged its further use. It is of note that, in Chandler's cases, where successful relief of the malignant course was achieved, vitreous loss usually occurred during the extraction. Although simple lens extraction has helped to save many eyes, the malignant glaucoma has often persisted when viireous loss did not occur.

The important role of the vitreous in phakic and aphakic malignant glaucomatous eyes was convincingly pointed out by Shaffer (1954), who described a posterior sclerotomy with a deep incision into the vitreous which enabled a release of vitreous posteriorly to soften the eye before lens extraction. In contrast to Chandler's successful cases, in which vitreous was lost anteriorly at the time of lens extraction, there was no loss of vitreous anteriorly in Shaffer's cases, and the malignant course frequently continued. Shaffer noted in two cases that, when attempts were made to re-form the anterior chamber with saline injection, it flowed into the posterior segment, leaving the eye hard with a persistent flat anterior chamber and with the vitreous and iris pressing forwards into the anterior segment under pressure. He recommended incision into the vitreous with a knife. In some cases in which the anterior hyaloid was incised, the malignant course was relieved, but in other cases it was necessary to make a deeper incision into the vitreous, piercing, he presumed, a detached posterior hyaloid membrane. Shaffer postulated that, in malignant glaucoma, aqueous was trapped in or behind a detached vitreous body in both phakic and aphakic eyes (see Figs I, 2, 3, opposite).

A new surgical approach was considered in 1964 , bearing in mind that the experiences of Chandler and Shaffer with lens extraction suggested that the vitreous played an important role in malignant glaucoma. As a working hypothesis it was considered that fluid (presumably aqueous humour) somehow accumulated under pressure in or behind the vitreous forcing the anterior hyaloid, lens, and ciliary processes forwards. The new approach was directed at the vitreous itself rather than at the lens in the hope of developing a simpler, safer, and more direct method. The rationale of this procedure was to release fluid trapped in or behind the vitreous, to open the anterior and posterior hyaloid membranes, and to re-form the anterior chamber, restoring the normal relationship of the anterior vitreous, lens, and ciliary body. It was hoped that this would prevent further the accumulation of fluid posteriorly and restore the normal fluid flow within the eye without 

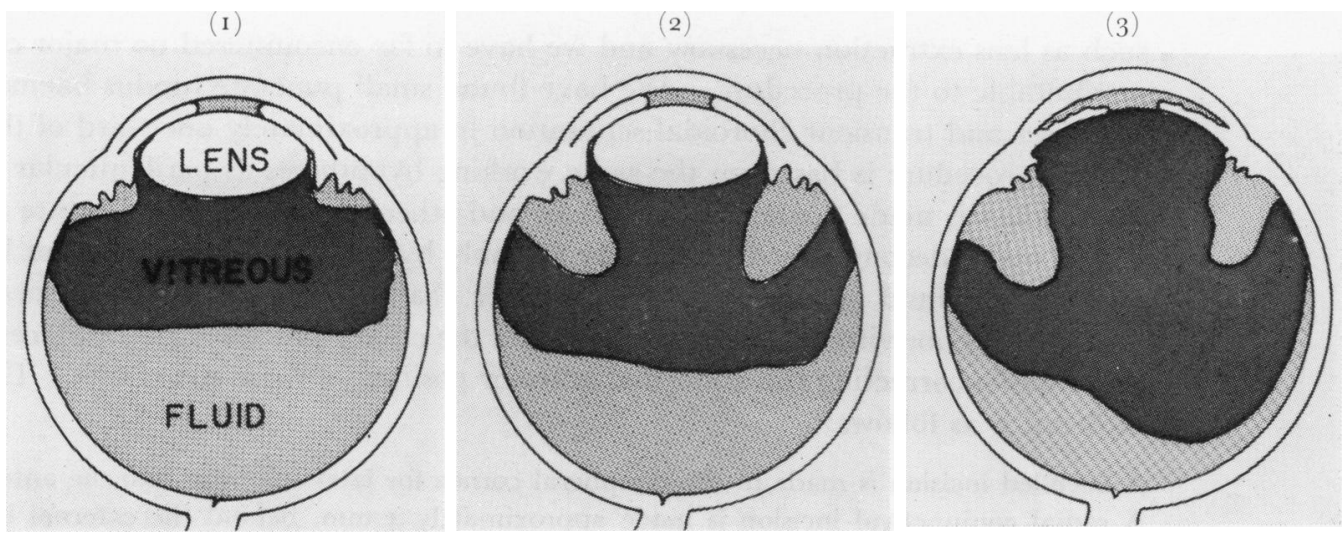

FIG. I Diagram of the working hypothesis of fluid trapped in or behind the vitreous body in a phakic eye with malignant glaucoma (Shaffer, 1954; Simmons, 1967, Chandler, 1967; Chandler and others, 1968)

FI G. 2 Same as Fig. I, but showing that fluid may according to the working hypothesis occur at various sites in the posterior segment. (Fluid could in fact be diffused throughout the vitreous but this is not shown by the diagram.)

FIG. 3 Same as above, but the eye is aphakic

removing the crystalline lens. Chandler (1964) gave a preliminary report of a series of cases in which he carried out this new procedure which we call perilenticular incision of the vitreous.

The operation consisted of an ab externo incision, approximately $7 \mathrm{~mm}$. long, which was made $2 \mathrm{~mm}$. parallel to the limbus. The ends of the incision were cut forwards to the limbus, making a small scleral flap. This flap was elevated, and an 18 gauge needle was then passed between the ciliary processes, through the zonule into the vitreous cavity, aiming the needle towards the optic nerve head. A clamp was placed on the needle $17 \mathrm{~mm}$. from the tip so that deeper penetration would not be possible. Fluid was allowed to escape from the needle and then the scleral wound was closed. Mydriatic-cycloplegic drops were then started and continued indefinitely. The anterior chamber was deepened to a normal depth by the injection of air.

Six eyes were treated in this manner by Chandler and the malignant course was relieved in all of them. Unfortunately, in those cases in which lens opacities were not already present, they rapidly formed afterwards. In one patient an inoperable retinal detachment developed. Therefore, although the malignant course was relieved in this series of cases, perilenticular incision of the vitreous carried such risk to the lens that it was abandoned in favour of a safer method. Nath ( I 966 ) reported the successful use of a similar but modified technique and Balakrishnan and Abraham (1969) reported its successful use in eight eyes for the relief of malignant glaucoma without complications. Experience with this procedure appears to demonstrate that removal of the lens itself is not necessary, and that other factors are responsible for the beneficial results of lens extraction and perilenticular incision of the vitreous.

In 1965 , in an attempt to relieve malignant glaucoma surgically without interfering with the lens, we devised, used, and began to teach a new procedure (Simmons, 1967; Chandler, 1967; Chandler and others, 1968). This consists of vitreous puncture and aspiration from a wound of entry $4 \mathrm{~mm}$. behind the limbus, followed by air injection into the anterior chamber with marked deepening of the latter. We have used this procedure successfully in 26 eyes, fourteen phakic and twelve aphakic. In one eye, it was necessary to repeat the procedure, and in the others a single operation sufficed. In no eye was additional surgery 
such as lens extraction necessary and we have so far encountered no major complications attributable to the procedure. We have found small punctate fundus haemorrhages in a few cases and transient choroidal separation in approximately one-third of the cases.

This procedure is based on the same working hypotheses as perilenticular incision, but the incision is made further from the lens and other details of the procedure are different.

The eye is decompressed posteriorly not only by insertion of a needle but by the actual aspiration of fluid or vitreous. The anterior chamber is markedly deepened with air so that the anterior vitreous body, the lens, and the ciliary processes are displaced backwards, presumably correcting the abnormal anterior position of these structures. The procedure in detail is as follows:

A bevelled incision is made in the peripheral cornea for later injection into the anterior chamber. A radial conjunctival incision is made approximately $4 \mathrm{~mm}$. behind the external limbus in both lower quadrants and a radial incision is made through the sclera a measured distance of $4 \mathrm{~mm}$. behind the external limbus into the suprachoroidal space in each lower quadrant (see Fig. 4). The wounds are opened to be sure that no fluid or blood exists in the supraciliary or suprachoroidal space since characteristically none is present in malignant glaucoma. The nasal scleral and conjunctival opening is then closed by suturing and is not used again. At the lower temporal opening, a ring of surface diathermy is placed around the scleral wound. A Wheeler knife (Fig. 5) is then passed into the vitreous cavity through the ciliary body, the knife being aimed towards the optic nerve to avoid contact with the crystalline lens. The wound is enlarged anteriorly and posteriorly to a total length in a radial direction of $3 \mathrm{~mm}$. with its centre $4 \mathrm{~mm}$. behind the external limbus. An 18 gauge needle (Fig. 6) is then passed a measured distance of $12 \mathrm{~mm}$. into the vitreous cavity in the direction of the optic nerve. Before the needle is inserted, a haemostat is placed on it at a point exactly $12 \mathrm{~mm}$. from the tip so that it cannot penetrate into the globe to a great depth. When the needle is fully inserted to this limit, the tip is moved from side to side a few millimetres, in an attempt to open more widely the pathway of the needle through the vitreous body and through the hyaloid membranes. Fluid is allowed to escape from the needle and wound spontaneously.

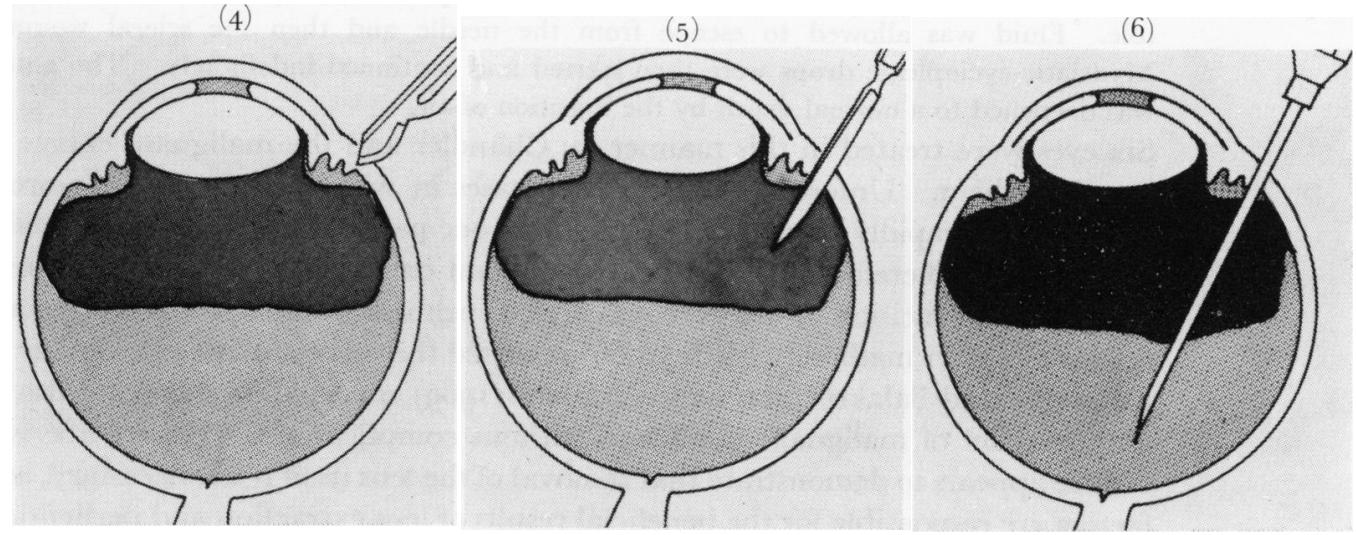

FIG. 4 Radial scleral incision $4 \mathrm{~mm}$. behind the external limbus for malignant glaucoma (see text). Diathermy (not shown) is then applied around the scleral wound

FIG. 5 Wheeler knife is used to pierce the uvea and enter the vitreous cavity. The knife is kept away from the lens by aiming it towards the optic nerve head (see text)

FIG. 6 An 18 gauge needle is inserted $12 \mathrm{~mm}$. into the eye (see text) (Haemostat guard to control needle depth not shown.) 
Whether fluid escapes or not, a syringe is attached to the needle (Fig. 7) and I to $\mathrm{I} \cdot 5 \mathrm{ml}$. is aspirated. (The fluid obtained is sometimes watery, and at other times it appears to be more viscous, like liquefied vitreous.)

A small amount of saline is injected into the anterior chamber through a needle partially to restore the shape of the semi-collapsed eye. The eye is not filled out to a completely normal shape and tension. (We avoid excessive saline injection into the anterior chamber at this time because in some cases saline will flow backwards into the eye and become trapped in or behind the vitreous body, and the eye will then again become firm, with anterior vitreous, the iris, and lens pushed tightly into the anterior segment as was the case before surgery. We have observed this phenomenon in several eyes as did Shaffer (1954) and for this reason we avoid extensive filling of the eye with saline.)
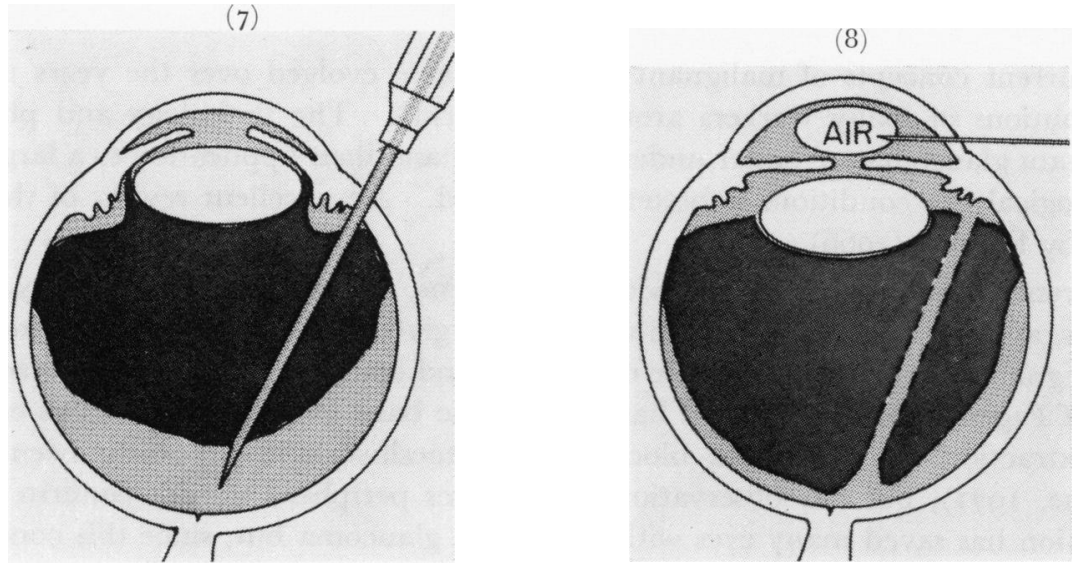

FIG. 7 A syringe is attached and I to I.5 $\mathrm{ml}$. of vitreous is aspirated (see text)

FIG. 8 A very large air bubble is placed in the anterior chamber to deepen it to abnormal depth (see text). The small air bubble shown will be enlarged by continued injection

The anterior chamber is then filled with a very large air bubble, artificially deepening the anterior chamber and pushing the iris, lens (when present), and anterior vitreous backwards into the eye. (In Fig. 8 the artist has represented the start of the air injection. The injection is continucd until the air bubble is much larger than drawn.) The anterior chamber is deepened to a depth greater than that of a normal myopic eye and much greater than the original depth of the shallow chamber usually encountered in cases of malignant glaucoma. The eye is thus left mushy-soft. Atropine is instilled and is continued regularly from the time of the operation for an indefinite period. In aphakic eyes with malignant glaucoma, the procedure is the same, except that the wound of entry is made at the limbus since there is no lens to be injured and the needle is passed through an existing or a newly made coloboma of the iris.

The patients on whom this procedure has been performed have been followed for from I to 6 years, and the eyes have remained healthy with continued use of atropine as stated above. Whether or not it is necessary to continue cycloplegics after this operation has not been fully established. In one case, however, we ceased the use of atropine and the malignant glaucoma did not recur during a I-year follow-up. In another case, atropine was discontinued and pllocarpine was later used for the residual glaucoma with no recurrence of the malignant glaucoma.

Since this operation has been uniformly successful in all fourteen phakic and twelve aphakic eyes on which we have tried it, and there have been no serious complications, 
we feel that it is a safe, reliable surgical procedure, and it has proved considerably simpler and safer in our hands than lens extraction. Even if a cataract is present in the eye at the time of the malignant glaucoma attack, we feel that it is safer to carry out this procedure first and to deal with the malignant glaucoma. Later, when the eye is quiet and the malignant glaucoma has been relieved, lens extraction can be undertaken in easier conditions should lens removal be required for vision.

In our opinion, the uniform success and safety of this operation make it at the present time the surgical treatment of choice for malignant glaucoma when a full trial of medical therapy has been unsuccessful.

\section{Discussion}

The current concepts of malignant glaucoma have evolved over the years through the contributions of many workers around the world $\ddagger$. The pathology and physiology of malignant glaucoma are better understood today and their application to a larger group of pathological eye conditions is better appreciated. An excellent review of the subject is given by Étienne (1966).

Current knowledge of the behaviour of the lens, zonules, and ciliary body, the role of miotics, and the importance of the vitreous has given us a better, broader understanding of malignant glaucoma. The role of the lens and zonule has been emphasized since the time of Pagenstecher (1877) and has become the basis for treatment of this condition by lens extraction. "Direct lens block" of the meshwork has recently been postulated (Levene, I971), but our observations of the lens periphery do not confirm this. Lens extraction has saved many eyes with malignant glaucoma but, since this condition often continues after lens extraction unless there is vitreous loss or unless there is incision of the vitreous body, the lens alone does not appear to be the primary key to malignant glaucoma. Relative laxness of the zonules, either spontaneous or induced by miotics, and even partial rupture of the zonules, was noted by Chandler and Grant (1962), who elucidated the role of miotics in precipitating or aggravating malignant glaucoma. Many excellent studies have demonstrated the shallowing of the anterior chamber and the forward movement of the lens with miotics. In normal eyes, in glaucomatous eyes with angleclosure, and in eyes with open-angle glaucoma, the application of this information is of great value*.

The relationship between the ciliary body, the vitreous face, and the lens appears to be an important factor. The ciliary processes show characteristic changes, including forward rotation, contact with the lens, sometimes flattening against the lens, and occasionally adherence to the lens. Lens apposition to the ciliary body was implicated as a feature of malignant glaucoma by Heuser (1877) who termed it incarceration of the lens by the ciliary body. The idea of a water-tight block between the lens and the ciliary body (Weiss and Shaffer, 1971) does not seem consistent with our observations; we have seen the tips of the ciliary processes in contact with the peripheral lens, but between the ciliary processes open spaces are distinctly visible. While the lens equator may be seen to touch the ciliary processes at all the points of the circumference where they can be observed in these eyes, we have never seen the lens in situ press the ciliary processes against the entire circumference so as to obliterate the spaces between the processes and block the flow of fluid. Therefore, although there is undoubtedly an abnormal relationship between the ciliary processes and the lens, it does not appear to us to be sufficient to block the forward flow of aqueous fluid.

¥ Kalfa, 1968; Daniele and Diotallevi, 1964; Hoshiwara, I964; Bisaria, 1965; Legrand, I968; Pillat, I967; Kurz, I966 * van Alphen, 1963; Jones and Watson, 1967; Wilkie, Drance, and Schulzer, 1969; Gorin, I966; Romano, I968; François, Goes, and Stockmans, I97I; Lowe, r970c; Bleeker, I96 I, r 963 
The vitreous appears to us to play a critical role. Behind the lens equator and ciliary processes in malignant glaucoma, the anterior vitreous face has been observed by us far forwards on the ciliary body, and in aphakic cases of malignant glaucoma it can be seen to touch or to adhere to the ciliary processes. In one aphakic case of malignant glaucoma reported by Shaffer (1954), a specimen of anterior vitreous pulled from the eye showed ciliary epithelium adhering to it, and so provided good histological evidence for abnormal contact between the ciliary body and the anterior vitreous. This abnormal relationship of the anterior vitreous, the lens periphery, and the ciliary processes may in some way cause a relative diversion of aqueous flow from its normal anterior route to a posterior route, allowing aqueous to accumulate in and behind the vitreous and thereby to push the anterior hyaloid, lens, and ciliary processes, forwards. The evidence for abnormal amounts of fluid in or behind the vitreous described above from slit-lamp examination of the vitreous and from surgical observations (Shaffer, 1954; Chandler, 1964) supports the hypothesis of the importance of the vitreous and the posterior segment in the pathophysiology of malignant glaucoma.

Finally, the fact that surgery of the vitreous body alone relieves malignant glaucoma suggests that the vitreous plays an important part in the pathophysiology of malignant glaucoma which cannot wisely be ignored. The mechanism by which the surgical procedure described above and currently recommended by us (Simmons, 1967; Chandler, 1967; Chandler and others, 1968) relieves the malignant glaucoma is not precisely known. Its efficacy may be due to the piercing of the vitreous body through the anterior and posterior hyaloid, thus providing a channel for forward flow of the posteriorly trapped aqueous as was the original rationale for the procedure. Grant's studies of enucleated human eyes have shown that the normal vitreous and normal anterior hyaloid face do not provide a significant resistance to aqueous flow. Therefore, if the openings made in the vitreous are significant, one must postulate that in malignant glaucoma some portion of the vitreous body, perhaps the anterior hyaloid and/or the posterior hyaloid, shows abnormal resistance to aqueous flow. While we and others (Hutchinson and Smith, 1967; Becker and Shaffer, 1965) have observed the anterior hyaloid membrane as being abnormally prominent and glassy-looking in cases of malignant glaucoma in aphakic eyes, we know of no evidence to support the concept of abnormal permeability of the hyaloid membrane.

The efficacy of the procedure may be due to the marked deepening of the anterior chamber with air which may break a relative block to the anterior flow of vitreous in the region of the anterior ciliary body or may correct a relative posterior diversion of aqueous in this region by correcting the abnormal apposition of the anterior vitreous face to the anterior ciliary body.

Recent information has shown that malignant glaucoma is not merely a condition that may follow surgery for angle-closure glaucoma. Current concepts include many pathological situations in the eye unrelated to angle-closure glaucoma. We and others have seen eyes behave in a fashion identical or nearly identical to that associated with malignant glaucoma in the absence of any evidence of primary angle-closure glaucoma and in cases of open-angle glaucoma (Rieser and Schwartz, 1971). In unoperated eyes and in eyes which have undergone intraocular surgery of various types unrelated to angleclosure glaucoma, features have been observed similar to those of malignant glaucoma (Levene, 197I). In aphakic eyes, as is well known, malignant glaucoma may persist after lens extraction for malignant glaucoma, but phenomena similar to those of malignant glaucoma have been noted after routine lens extractions in eyes with no apparent preoperative abnormality other than cataract (Hutchinson and Smith, 1967). Whether we 
call these cases of malignant glaucoma, using the old term in a new broader sense, of whether we devise a new terminology to describe such phenomena should perhaps await. further experimentation.

\title{
Conclusion
}

Many questions still remain in regard to malignant glaucoma, and it is to be hoped that future observations will gradually elucidate them. Today, however, medical therapyes consisting of the concurrent use of mydriatic-cycloplegic drops, carbonic anhydras $\sigma_{0}$ inhibitors, and hyperosmotic agents is available, and is proving to be effective in half thecases when continued for 5 days. In addition, the vitreous surgery described above is in our opinion a safe and reliable surgical procedure in cases of malignant glaucoma whiclo. are not relieved by medical therapy.

\section{COMMENTARY}

MALIGNANT GLAUCOMA

Chandler's anterior chamber deepening procedure with gonioscopy used for diagnosis of the extent of synaechial closure of the angle does not seem to predispose to malignant glaucoma.

Even though it is known that there is a predisposition to malignant glaucoma in the fellow eye $\stackrel{?}{?}$ this eye should always be treated with a peripheral iridectomy as early as possible after the onset of malignant glaucoma in the opposite eye. No medical treatment of any sort should be given beforđ the peripheral iridectomy, since both miotics and mydriatics may be dangerous before surgery is performed. If the fellow eye has been operated upon when the angle has been completely open, then malignant glaucoma has not occurred. However, if angle-closure is present in the fellow eye at the time of surgery, then malignant glaucoma becomes very common. Malignant glaucoman sometimes follows routine cataract extraction.

\section{Medical control of the glaucomas}

\author{
KENNETH T. RIGHARDSON \\ Department of Ophthalmology, University of Pittsburgh School of Medicine, and Eye and Ear \\ Hospital, Pittsburgh, Pennsylvania 152 I 3 , U.S.A.
}

The principles of medical therapy in chronic glaucoma are straightforward once the physician has determined the pressure level required for adequate control. This pressure level must, of course, relate to the particular patient and must not be simply $\stackrel{?}{+}$ some average pressure arrived at by manipulating random population statistics. It is impossible to set rigid guidelines for achieving adequate pressure control but, in general, these should relate to the "functional status" of the patient, since the need for more $\frac{\text { ? }}{\mathbb{8}}$ consistent and lower pressure control increases as his functional status deteriorates. Guide $-\frac{\Omega}{0}$ lines for pressure control may thus be roughly related to functional status (Figure, opposite). 